\title{
Applications of Mobile GIS in Forestry South Australia
}

\author{
D. T. Battad ${ }^{\text {a }}$ P. Mackenzie ${ }^{\text {b }}$ \\ ${ }^{\text {a }}$ South Australian Forestry Corporation, Mt. Gambier, South Australia, Australia 5290 - \\ battad.dionisio@forestrysa.com.au \\ b South Australian Forestry Corporation, Mt. Gambier, South Australia, Australia 5290 - \\ mackenzie.peter@forestrysa.com.au
}

ICWG V/I: Land-Based Mobile Mapping Systems

KEY WORDS: mobile GIS, GIS, GPS, wetlands, mapping, forest health, forestry

\begin{abstract}
South Australian Forestry Corporation (ForestrySA) had been actively investigating the applications of mobile GIS in forestry for the past few years. The main objective is to develop an integrated mobile GIS capability that allows staff to collect new spatial information, verify existing data, and remotely access and post data from the field. Two (2) prototype mobile GIS applications have been developed already using the Environmental Systems Research Institute (ESRI) ARCGIS ${ }^{\mathrm{R}}$ technology as the main spatial component. These prototype systems are the Forest Health Surveillance System and the Mobile GIS for Wetlands System.
\end{abstract}

The Forest Health Surveillance System prototype is used primarily for aerial forest health surveillance. It was developed using a tablet PC with $\operatorname{ArcMap}^{R}$ GIS. A customised toolbar was developed using ArcObjects ${ }^{R}$ in the Visual Basic 6 Integrated Development Environment (IDE). The resulting dynamic linked library provides a suite of custom tools which enables the following:

- $\quad$ quickly create spatial features and attribute the data

- full utilisation of global positioning system (GPS) technology

- $\quad$ excellent screen display navigation tools, i.e. pan, rotate map, capture of flight path

- $\quad$ seamless integration of data into GIS as geodatabase (GDB) feature classes

- $\quad$ screen entry of text and conversion to annotation feature classes

The Mobile GIS for Wetlands System prototype was developed for verifying existing wetland areas within ForestrySA's plantation estate, collect new wetland data, and record wetland conditions. Mapping of actual wetlands within ForestrySA's plantation estate is very critical because of the need to establish protection buffers around these features during the implementation of plantation operations. System development has been focussed on a mobile phone platform (HTC HD2 ${ }^{\mathrm{R}}$ ) with Windows ${ }^{\mathrm{R}}$ Mobile 6, ESRI's ArcGIS $^{R}$ Mobile software development kit (SDK) employing ArcObjects ${ }^{R}$ written on C\#.NET IDE, and ArcGIS Server ${ }^{R}$ technology. The system is also implemented in the VILIV ${ }^{\mathrm{R}}$ X70. The system has undergone testing by ForestrySA staff and the refinements had been incorporated in the latest version of the system. The system has the following functionalities:

- display and query strategic data layers

- $\quad$ collect and edit spatial and attribute data

- full utilisation of global positioning GPS technology

- distance and area measurements

- display of high resolution imagery

- $\quad$ seamless integration of data into GIS as feature classes

- $\quad$ screen display and navigation tools, i.e. pan, zoom in/out, rotate map

- capture of flight path

The next stages in the development of mobile GIS technologies at ForestrySA are to enhance the systems' capabilities as one of the organization main data capture systems. These include incorporating other applications, e.g. roads/tracks mapping, mapping of significant sites, etc., and migration of the system to Windows Phone7.

\section{INTRODUCTION}

Mobile GIS involves the integration of GIS technology with a variety of other technology which includes Global Positioning Systems (GPS), wireless communication and database technology. It includes accessing, using and maintaining geospatial data directly in the field (McLarin 2004).

Beginning in the 1990s, there was a rapid development in mobile mapping applications technology (Sarjakoski and Sarjakoski 2007). The appearance of mobile computing devices and cheap location sensing devices has made location information become an important resource for both mobile and desktop users (Leonhardt et al 1996).

The traditional methods of field data collection and making edits/updates using paper maps are time-consuming. These practices are prone to numerous errors. Mobile GIS enables staff to bring out spatial data in electronic format into the field, capture data and/or make the necessary edits to the data, and post the data into the enterprise GIS database in near real-time. 
Mobile GIS is made possible with developments in GPS technology, rugged hand-held computing technologies, wireless communications, and GIS software for mobile platforms (McLarin 2004, Poorazizi et al 2008, Schanchun et al 2004). The provision of up-to-date information, analysis and better decision-making is made possible.

ForestrySA had recognized the potentials of mobile GIS technology for forest resource management and planning, particularly on forest plantation data collection, resource survey and monitoring of operational activities. Since 2008, ForestrySA started investigating the implementation of mobile GIS. Mobile GIS extends GIS technology beyond the office and allows accurate, real-time business decisions and collaborations in both field and office environments (ESRI 2007).

The development of mobile GIS capabilities in ForestrySA provides many advantages to the organization. This leads to improved efficiency and better accuracy in the collection, use and dissemination of spatial information by field staff. Data can be made available to other staff by enabling remote access to most current and accurate information. New data collected are more accurate through reduced data handling. The development of customised tools and automated processes can facilitate the preparation of spatial data for mapping and reporting purposes.

\section{DESCRIPTION OF THE PROTOTYPE SYSTEMS}

ForestrySA had developed two (2) of mobile GIS application prototypes under 2 different platforms, namely: Forest Health Monitoring System, and Wetlands Assessment System.

\subsection{Forest health monitoring system}

This system was developed because of the need for a capability to undertake aerial observations and assessments of the location and impacts of various insect infestations on ForestrySA plantations, particularly Esigella californica, Sirex wasp (Sirex noctillio).

The functional and data requirements of this mobile GIS system and the appropriate platform were identified after a series of meetings/consultations with the Principal Research Scientist Forest Health in ForestrySA.

The system was developed using a tablet computer with Windows 7 operating system and $3 \mathrm{G}$ connection. The system is GPS-enabled using a Bluetooth GPS device (Garmin ${ }^{\mathrm{R}}$ GPS 10).

The system is designed mainly to capture locational data related to insect infestations. It can also be used to capture data regarding location of lightning strikes, dead or dying trees (Figure 1).

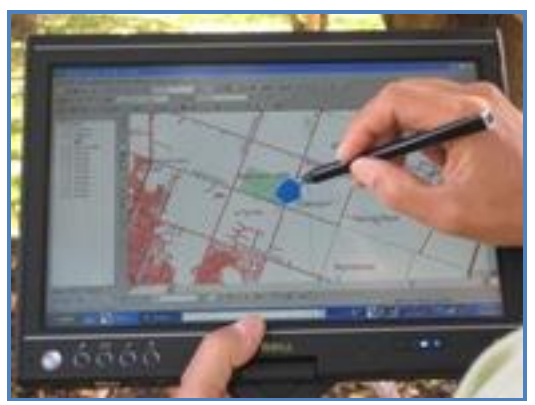

Figure 1. Data capture with the Forest Health Monitoring system

The system has the following functional capabilities such as map display, mapping and editing of features, etc. Figure 2 shows a list of the functionalities of the system.

\begin{tabular}{|c|c|c|}
\hline \multicolumn{3}{|l|}{ Forest Health Surveillance } \\
\hline Button & Function & Description \\
\hline 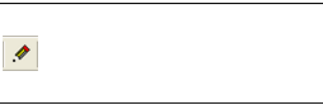 & $\begin{array}{l}\text { Collect features by } \\
\text { polygon }\end{array}$ & $\begin{array}{l}\text { Draw a polygon on } \\
\text { the screen, to close } \\
\text { and code, click a } \\
\text { "code feature" button }\end{array}$ \\
\hline$x$ & Delete Features & $\begin{array}{l}\text { Delete either point or } \\
\text { polygon features - } \\
\text { click then drag a box } \\
\text { over the features you } \\
\text { wish to remove. }\end{array}$ \\
\hline$\angle$ & Write text on screen & $\begin{array}{l}\text { Write text on the } \\
\text { screen with stylus. }\end{array}$ \\
\hline \begin{tabular}{|l|l|l|l|l|l} 
Sirex & Aphids & Dead & Dying Lightning & Other \\
\end{tabular} & Code feature. & Used to code features. \\
\hline Convert Text & $\begin{array}{l}\text { Convert text to } \\
\text { feature class } \\
\text { annotation }\end{array}$ & $\begin{array}{l}\text { Click to convert text } \\
\text { on screen to } \\
\text { annotation feature } \\
\text { class. }\end{array}$ \\
\hline \# & $\begin{array}{l}\text { Collect features by } \\
\text { point }\end{array}$ & $\begin{array}{l}\text { Add point(s), to close } \\
\text { and code click a "code } \\
\text { feature" button. }\end{array}$ \\
\hline GPS & Start GPS & Start GPS \\
\hline Comms Port & $\begin{array}{l}\text { Communication port } \\
\text { GPS }\end{array}$ & $\begin{array}{l}\text { Select the } \\
\text { communication port } \\
\text { the GPS is } \\
\text { transmitting from. }\end{array}$ \\
\hline Rotate off & $\begin{array}{l}\text { Toggle to rotate map } \\
\text { on/off. }\end{array}$ & $\begin{array}{l}\text { Rotate on - the map } \\
\text { will rotate with the } \\
\text { direction of travel. } \\
\text { Rotate off - the map } \\
\text { stops rotating. }\end{array}$ \\
\hline Log Track off & $\begin{array}{l}\text { Toggle to log track } \\
\text { on/off }\end{array}$ & $\begin{array}{l}\text { When turned on - } \\
\text { Logs the flight path } \\
\text { and stores it as a } \\
\text { shapefile. }\end{array}$ \\
\hline$\phi$ & Centres the map & $\begin{array}{l}\text { Centres the map to } \\
\text { current position }\end{array}$ \\
\hline 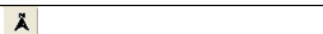 & Returns map to north & Returns map to north \\
\hline
\end{tabular}

Figure 2. Functionalities of the Forest Health Monitoring System

Cached spatial data from the corporate database is preloaded into the system. The data is used as a reference for the capture of health-related information.

Captured data is downloaded and provided to operations staff for ground verification and to collect more detailed information, if necessary.

Once the data is incorporated into the corporate database, it is accessible to other users through the Forest Records Information System (FRIS), another ForestrySA GIS-based system (Figure 3).

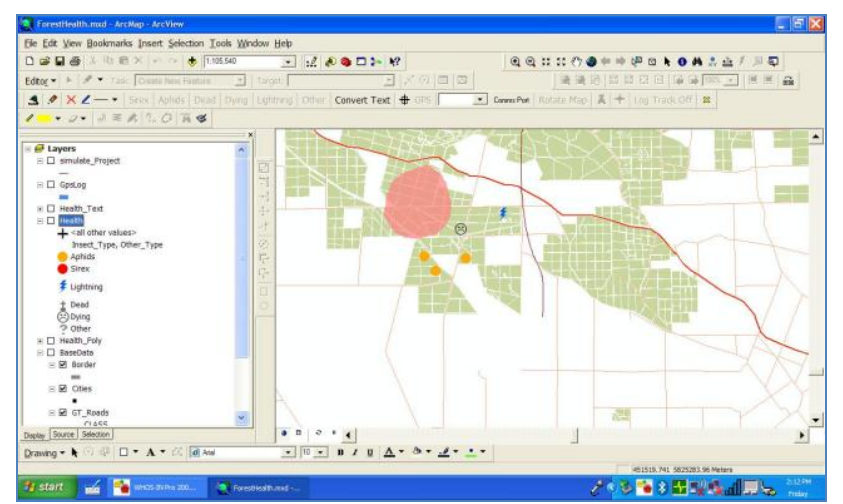

Figure 3. Display of captured data 
There is no intention to pursue further development of the system.

\subsection{Wetlands assessment system prototype}

The geology of the Limestone Coast of South Australia has resulted to the development of a wetland complex. These wetlands and their associated vegetation are recognized and managed for their conservation values.

The wetlands assessment mobile GIS system prototype was developed in order that field staff can verify the location, determine the boundaries, and assess the conditions of wetlands within ForestrySA plantations. There is a need to undertake these verifications and assessments because of the requirements for the applications of conservation buffers around these wetlands. Likewise, there are also influences on the implementation of plantation maintenance activities like herbicide spraying, fertilizer applications, etc. The conditions of the wetlands and the required protection buffers have a significant impact on the extent of productive forest plantations.

The system is envisioned to evolve as one of the mainstream data capture systems for ForestrySA, e.g. mapping of tracks and trails, firebreaks, stocking, fertilizer and herbicide applications, etc.

The system is built on the HTC HD2 ${ }^{\mathrm{R}}$ mobile phone. Several platforms are currently available implementing smartphonebased mobile surveys. This a great development as organisations which implement these solutions can choose from a number of technologies in order to suit their needs and fit their existing infrastructure (Feygin 2011).

The technology is based on ArcGIS ${ }^{\mathrm{R}}$ Mobile SDK and utilises ArcServer $^{R}$ technology from ESRI so it does not require additional software licensing. It also uses built-in GPS (Figure 4).

The first stage in system development was the identification and assessment of suitable platfoms. A user needs analysis was undertaken through a series of workshops involving the different groups within ForestrySA. From the workshops, a list of desired functional requirements was generated.

The HTC HD2 ${ }^{\mathrm{R}}$ smart phone was identified as the most appropriate platform for development because ForestrySA has already procured the phones for the staff. The system is implemented using ESRI's $\operatorname{ArcGIS}^{\mathrm{R}}$ Mobile software development kit (SDK) which employs ArcObjects ${ }^{\mathrm{R}}$ on C\#.NET IDE, and ArcGIS Server ${ }^{R}$ technology (Figure 4). It also uses built-in GPS. The same system is also implemented using the VILIV $X 70^{R}$ for users who require a bigger display screen (Figure 5).

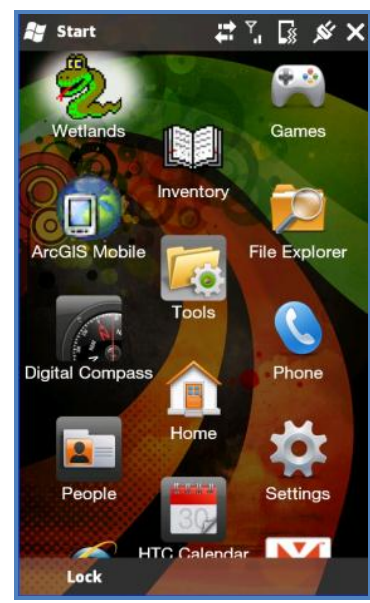

Figure 4. Illustration of the wetlands mobile GIS interface with the HTC HD2 mobile phone.

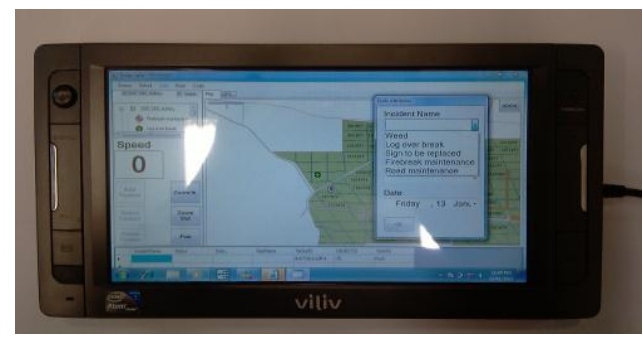

Figure 5. Illustration of the wetlands mobile GIS interface with the VILIV X70.

2.2.1 System functionalities: The prototype system implements specific functionalities that were identified by the users from the user needs analysis stage.

- Definition of region of interest: ForestrySA's plantation estates are usually identified by regions, namely: Green Triangle (covers the entire south east including Victoria); Mount Lofty Ranges (covers the Adelaide Hills and Fleurieu Peninsula areas); and Mid-North (covers the Bundaleer and Wirrabarra areas). The system enables the user to define the region of interest for data display and other spatial operations (Figure 6). This functionality defines the default extent of data display.

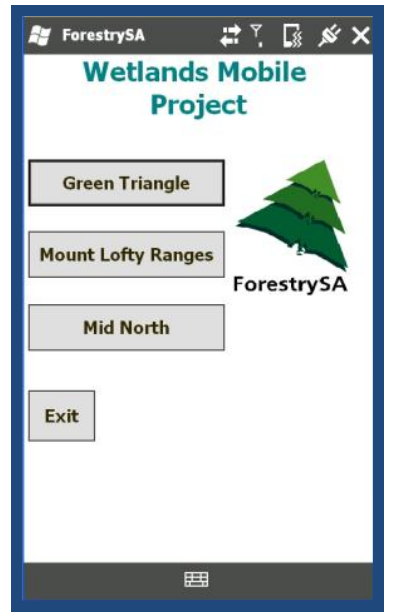

Figure 6. System interface for defining region of interest 
- Map display and navigation tools: The relevant base data for the region of interest is displayed. Several map navigation tools are enabled for navigating on the map display (Figure 7). The user also has the option to add other datasets to the display, e.g. images (Figure 8).
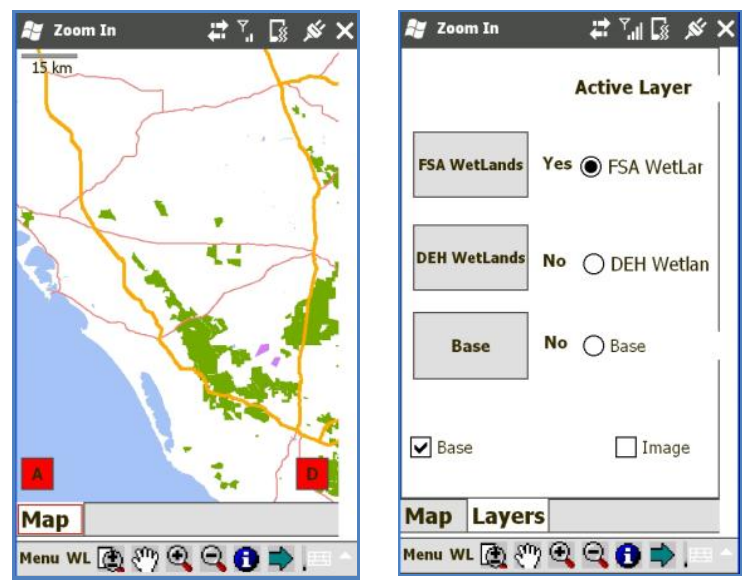

Figure 7. Map display and navigation tools

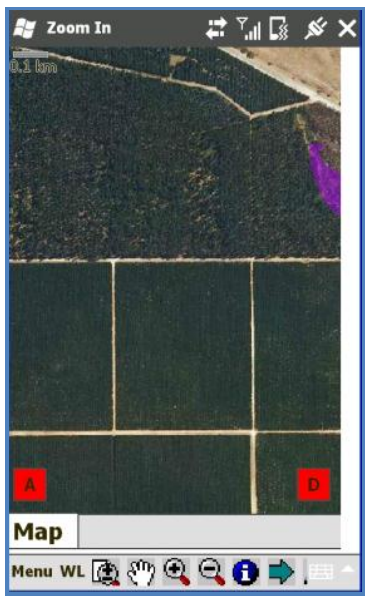

Figure 8. Display of images

- Identify function: This functionality enables the user to query a feature and display its associated attributes.

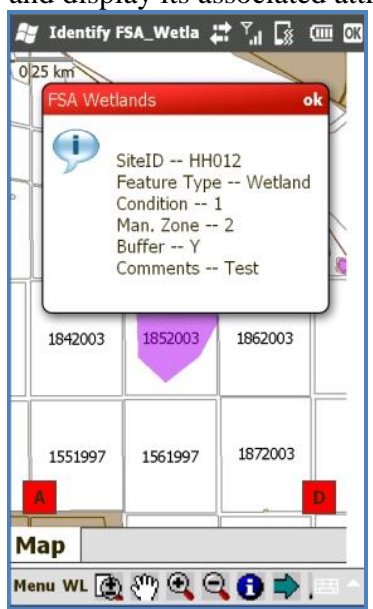

Figure 7. Identification of features

- Mapping wetland boundary: With the built-in GPS system, the user can use the sketch tools to capture the boundaries of any wetland or area of interest. The user can also enter the attributes for a feature using a predefined attribute entry template. The user can then save the data as a new feature.
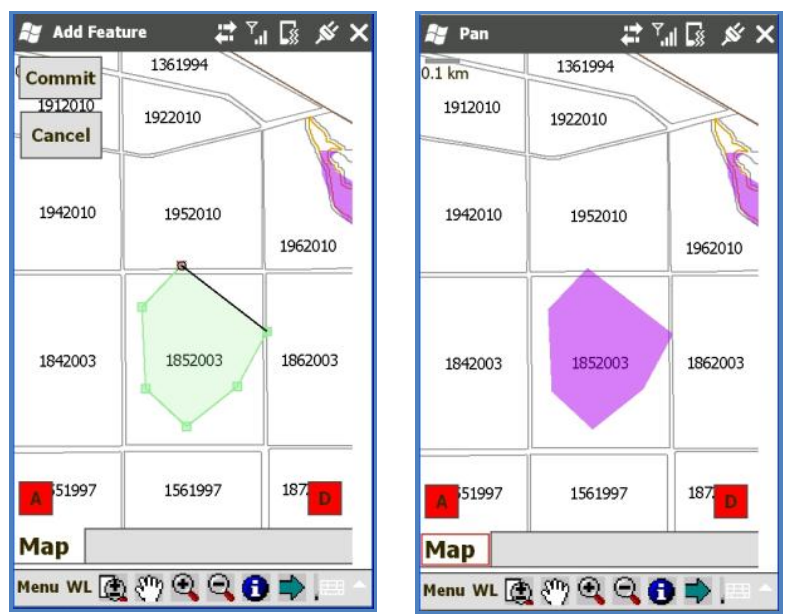

Figure 8. Mapping of wetland boundaries

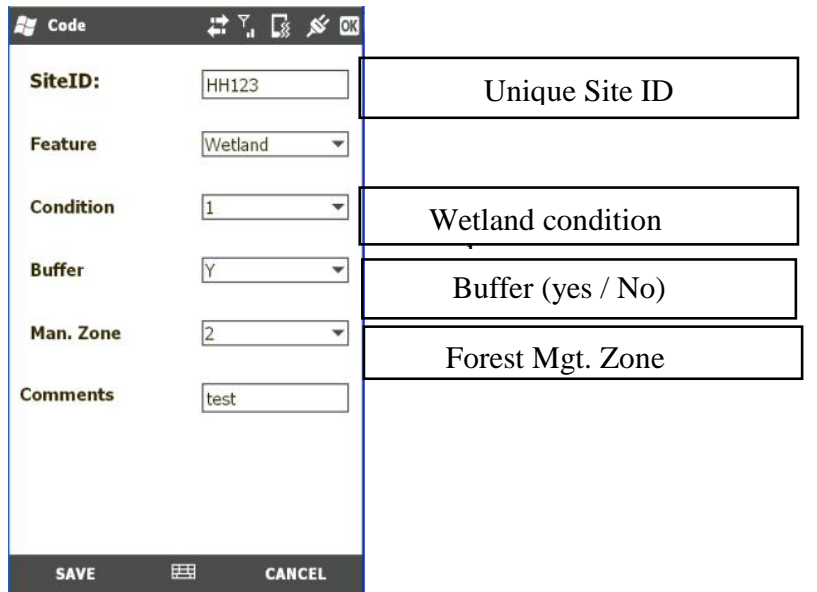

Figure 9. Template for coding attributes of features

- Editing wetland boundaries: The system also enables the user to edit and update the boundaries of existing wetlands. The new boundaries can then be downloaded and used to update the corporate dataset. 


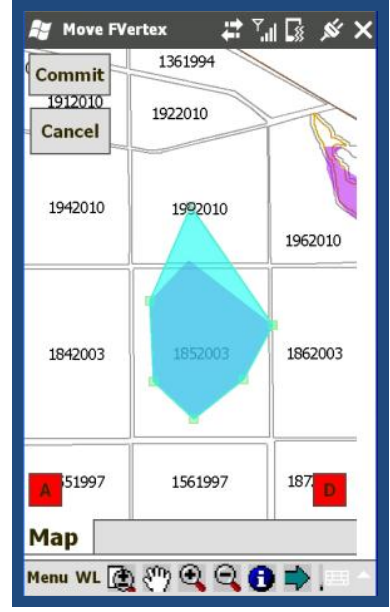

Figure 10. Editing geometries of existing features

- Area and distance measurements: Areas of polygon or length of line features surveyed/mapped in the field can also be estimated using this functionality. This enables the user to immediately determine changes in the extent or length of a feature.
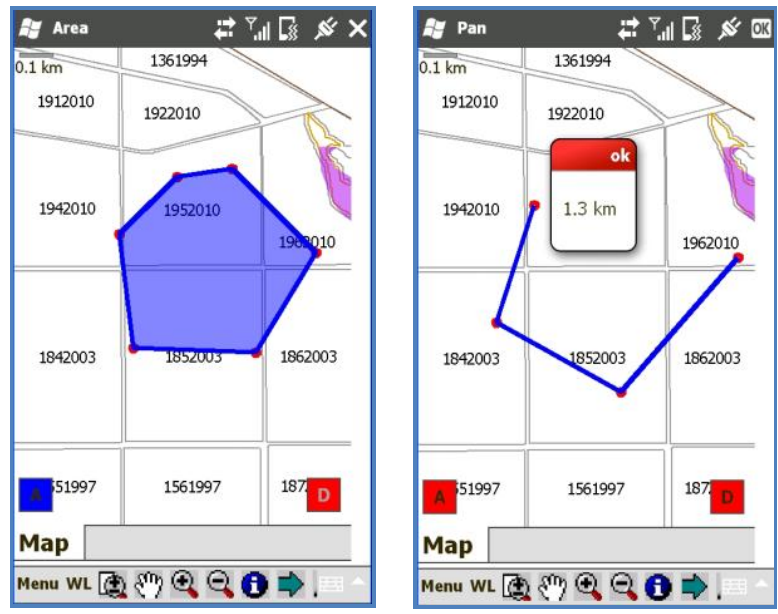

Figure 11. Area and distance measurement functions

- Post data to server: It is envisioned that spatial data collected/updated using the system can be posted remotely to corporate database in areas where there are WI-FI connection through system synchronisation. This is not yet operational. Currently, users download the data from the mobile phone to the GIS manually.

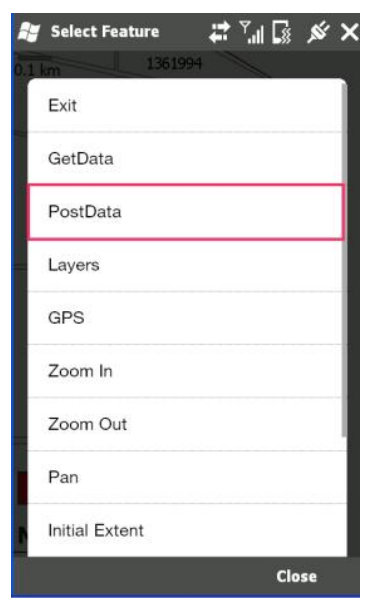

Figure 12. Posting data remotely to the server

\section{CONCLUSIONS}

These prototype systems are still being refined but they already demonstrate how ForestrySA is trying to pursue one of the main GIS development directions - the development of automated data capture and data update capabilities using mobile GIS technology. The system will improve efficiency as well as minimize risks from errors due to data handling. Mobile GIS is one of the building blocks for the strategic development of GIS capabilities in ForestrySA. It is planned to be the main spatial data capture and update technology used by field staff.

The next stages, after implementation of the required refinements and prior to operational deployment of the system, are the implementation of several training sessions for the users and system documentation. The system shall also be migrated to the Windows Phone 7 platform.

\section{REFERENCES}

McLarin, P., 2004. Mobile GIS technology update. http://www/gisdevelopment.net/technology/mobilemapping/ma 04160pf.htm.

ESRI, 2007. Mobile GIS.

http://www.esri.com/technology-topics/mobile-gis/index.html.

ESRI (Environmental Systems Resource Institute). 2008. ArcGIS. ESRI, Redlands, California.

ESRI (Environmental Systems Resource Institute). 2008. ArcGIS Server. ESRI, Redlands, California.

Feygin, S., 2011. Data for the People, by the People: Designing Smartphone-based GISystems for Environmental resource Assessment.

http://gislounge.com/data-for-the-people-by-the-peopledesigning-smartphone-based-gisystems-for-environmentalresource-assessment/

Garmin GPS 10. http://www.garmin.com/au/

HTC HD2. http://www.htc.com/us/products/t-mobile-hd2

Leonhardt, U., J. Magee, and P. Dias, 1996.Location service in mobile computing environments. Comput. Graphic., 20(5):627632 . 
Poorazizi, E.,A.A. Alesheikh and S. Behzadi, 2008. Developing a Mobile GIS for Field Geospatial Acquisition. Journal of Applied Sciences,8:3279-3283.

http://scialert.net/fulltext/?doi=jas.2008.3279.3283.

Sarjakowski,T. and Sarjakowski L.T., 2007. A Real-time Generalisation and Map Adaptation Approach for LocationBased Services. In: A.M. William, R. Anne and L.T. Sarjakowski (eds) Generalisation of Geographic Information, Elsevier Science B.V., Amsterdam, pp. 137-159.

Schanchun, C. and S. Liangping, 2004. Designing a Real-Time Mobile GIS. Proc. Mapasia2004, Beijing, China, 26-29 Aug. http://www/gisdevelopment.net/technology/mobilemapping/ma 04088pf.htm.

VILIV X70 http://www.myviliv.com/

\section{ACKNOWLEDGEMENT}

The authors acknowledge Dr. Jim O'Hehir whose support and encouragement made possible the implementation of this project.

We also acknowledge the contributions of the C\&R Rangers, Bryan Haywood and Troy Horn, for helping us identify the functional requirements and for testing the prototype system. 